Molecule Statistical Thermodynamics Simulation of Nanoindentation of Single Crystal Copper with EAM Potential

This article has been downloaded from IOPscience. Please scroll down to see the full text article.

2011 Chinese Phys. Lett. 28046201

(http://iopscience.iop.org/0256-307X/28/4/046201)

View the table of contents for this issue, or go to the journal homepage for more

Download details:

IP Address: 159.226.231.78

The article was downloaded on 21/03/2012 at 08:38

Please note that terms and conditions apply. 


\title{
Molecule Statistical Thermodynamics Simulation of Nanoindentation of Single Crystal Copper with EAM Potential*
}

\author{
TAN Hao(谭浩 $)^{1,2}$, WANG Hai-Ying(汪海英 $)^{1 * *}$, XIA Meng-Fen(夏蒙棼 $)^{1}$, KE Fu-Jiu(柯孚久 $)^{3}$, \\ BAI Yi-Long(白以龙 $)^{1}$ \\ ${ }^{1}$ State Key Laboratory of Nonlinear Mechanics (LNM), Institute of Mechanics, Chinese Academy of Sciences, \\ Beijing 100190 \\ ${ }^{2}$ Graduate University of Chinese Academy of Sciences, Beijing 100049 \\ ${ }^{3}$ School of Physics and Nuclear Energy Engineering, Beijing University of Aeronautics and Astronautics, Beijing 100191
}

(Received 13 January 2011)

\begin{abstract}
The quasistatic nanoindentation process of a spherical indenter in a single crystal copper is investigated with the molecular statistical thermodynamics (MST) method based on the embedded atom method (EAM) potential. The indentation modulus obtained in the MST simulation is $129.9 \mathrm{GPa}$, which agrees well with the theoretical prediction $(129 \mathrm{GPa})$. In the elastic regime, the obtained maximum displacement of the indenter is two times the contact depth and the contact area is qualitatively proportional to the contact depth, which agrees well with Hertzian elastic theory of contact. The MST simulation can reproduce the nucleation of dislocation as well. Moreover, the efficiency of the MST method is about 8 times higher than that of traditional MD simulations.
\end{abstract}

PACS: 62.20 F-, 62.25.-g DOI: $10.1088 / 0256-307 \mathrm{X} / 28 / 4 / 046201$

Nanoindentation is a powerful tool to investigate the nano- and micro-scale mechanical properties of materials, and has been widely used in material and mechanical engineering. ${ }^{[1,2]}$ Due to the inherent complexity of the nanoindentation process, complementary molecular dynamics (MD) simulations have been carried out by many researchers in order to assess the dislocation nucleation mechanism and propagation during indentation. ${ }^{[3,4]}$ An appealing feature of MD is that it follows the actual dynamical evolution of all atoms. However, for realistic systems, it is practically impossible to track all atoms by solving the equations of motion. ${ }^{[5]}$ More importantly, to resolve the individual motion of atoms requires a time step of approximately femtoseconds. Hence, the length and time scales that can be probed using MD are still fairly limited, even on the most advanced massively paralleled computers.

To manage the computational burden and establish a reliable computational scheme, $\mathrm{Hu}$ and Wang et $a l^{[6-8]}$ presented an inter-atomic potential based method, the molecular statistical thermodynamics (MST) method, to simulate the quasi-static deformation of materials at finite temperature. However, as a prototype study, the simulation in Ref. [8] is based on the Lenard-Johns potential, which has deficits in modeling metallic matrials. In this Letter, the MST method simulation of quasi-static nanoindentation based on embedded atom method (EAM) is performed on a single crystal copper under nanoindentation. We compare the data obtained for simulation in the elastic stage with the Hertzian elastic theory of contact. Also, the dislocation structure and the computational cost are analyzed.

The basic idea of the MST method is particleoscillator duality, that is, we treat atoms as particles with determined equilibrium positions when we examine the mechanical deformation of the atomic lattice; while treating atoms as oscillators with various frequencies when we examine the influence of the thermal oscillations of atoms on mechanical deformation. By assuming the oscillators to be local harmonic ones, the Helmholtz free energy ${ }^{[9,10]}$ of a solid can be represented as

$$
A=\Phi+3 k T \sum_{i=1}^{N} \ln \left(\frac{\hbar\left|\boldsymbol{D}_{i}\right|^{1 / 6}}{k T}\right),
$$

where $\Phi$ is the static lattice energy (potential energy), $T$ is the temperature, $k$ is the Boltzmann constant, $\hbar$ is the Planck constant, $N$ is the total number of atoms in system, $\left|\boldsymbol{D}_{i}\right|$ is the determinant of the local dynamical matrix of atom $i$, whose element is defined as

$$
D_{i}^{\zeta \eta}=\frac{1}{m_{i}} \frac{\partial^{2} \Phi}{\partial x_{i \zeta} \partial x_{i \eta}},
$$

where $m_{i}$ is the mass of atom $i$, and $x_{i \zeta}$ is the $\zeta$ th coordination of atom $i$.

We assume that the indenter is a rigid sphere, whose interaction with atoms in the sample can be represented by a repulsive potential as: ${ }^{[11]}$

$$
V= \begin{cases}A\left(R_{0}-r\right)^{3}, & \text { for } \quad r<R_{0}, \\ 0, & \text { for } r \geq R_{0}\end{cases}
$$

*Supported by the National Natural Science Foundation of China under Grant Nos 10932011, 10772181, 11021262, 10732090 and 10772012, and the National Basic Research Program of China under Grant No 2007CB814803.

**Email: why@lnm.imech.ac.cn

(C) 2011 Chinese Physical Society and IOP Publishing Ltd 
where $A$ is a force constant, $R_{0}$ is the radius of the spherical indenter and $r$ is the distance from the centre of atom to the center of the spherical indenter. In our simulation, $A=3.3084 \mathrm{eV} / \AA^{3}, R_{0}=15 \AA$.

In this simulation, the sample is single crystal copper with dimensions $75.9 \times 75.9 \times 61.5$, containing 32358 atoms. The top surface of the sample is the (001) crystal plane and the other side surfaces are (100) and (010) planes, respectively. The top surface is traction free, while two atom layers at both the bottom and the side surfaces are fixed. The temperature is kept constant at $300 \mathrm{~K}$ during the entire simulation time.

To compute the potential energy of copper, the embedded atom method (EAM) developed by Daw and Baskes $^{[12]}$ is employed,

$$
\Phi=\frac{1}{2} \sum_{i j} V\left(\mathbf{r}_{i j}\right)+\sum_{i} F_{i}\left(\sum_{i \neq j} \rho_{j}\left(\mathbf{r}_{i j}\right)\right),
$$

where $\boldsymbol{r}_{i j}$ is the distance between atom $i$ and atom $j$, $V$ is a pair-wise potential as a function of $\boldsymbol{r}_{i j}, \rho\left(\boldsymbol{r}_{i j}\right)$ is the electron density introduced by atom $j$ at the site of atom $i$, and $F_{i}$ is the embedding energy of atom $i$ as a function of atomic electron density $\rho$. In fact, $V$, $F_{i}$ and $\rho_{i}$ are fitting functions based on actual experimental dates, and different researchers may obtain different fitting functions. EAM views each atom as embedded in a host lattice consisting of all other atoms, which permits calculations employing an electron density. Since the electron density is a summation over many atoms, usually limited by a cutoff radius, the EAM potential is a multibody potential. An empirical potential for copper, developed by Mishin et al., ${ }^{[13]}$ was used in the simulation.

We first compare the results from the MST simulation to the solution of Hertzian elastic contact theory for a spherical indenter. In Hertzian elastic contact theory, the relationship between applied force $P$ and indenter displacement $h$ is ${ }^{[14]}$

$$
P=\frac{4}{3} E^{*} R^{1 / 2} h^{3 / 2},
$$

where $R$ is the curvature radius of the indenter and $E^{*}$ is the reduced modulus. For elastically anisotropic materials, Vlassak and Nix have shown that the releationshp in Eq. (5) still holds, providing that the reduced modulus is re-defined as ${ }^{[15,16]}$

$$
E^{*}=\left(\frac{1}{E}-\frac{1-\nu_{i}^{2}}{E_{i}}\right)^{-1}
$$

where $E$ is the "indentation modulus", which is related to the elastic constants of the sample in different directions, $E_{i}$ and $\nu_{i}$ are Young's modulus and Poisson's ratios of the indenter. In the case of a perfectly rigid indenter, $E^{*}$ reduces to the indentation modulus $E$.
The force vs indenter displacement data obtained from the MST simulation is plotted as round solid dots in Fig. 1. Noticeably, the force drops abruptly at the displacement about $5.4 \AA$ (from point $\mathrm{B}$ to point $\mathrm{C}$ ), which indicates dislocation nucleation and the onset of plasticity. We fit the data from point A to point $\mathrm{B}$ with the solution of the Hertzian elastic contact theory (Eq. (5)) and obtain the indentation modulus of the sample as $129.9 \mathrm{GPa}$, which agrees well with the results of Vlassak and Nix (129 GPa for theoretical prediction and $124 \mathrm{GPa}$ for experiments). [15,16] With $E=129.9 \mathrm{GPa}$, we draw the theoretical curve of Eq. (5) in Fig. 1. Obviously, before dislocation nucleation, the simulation data overlap with the curve.

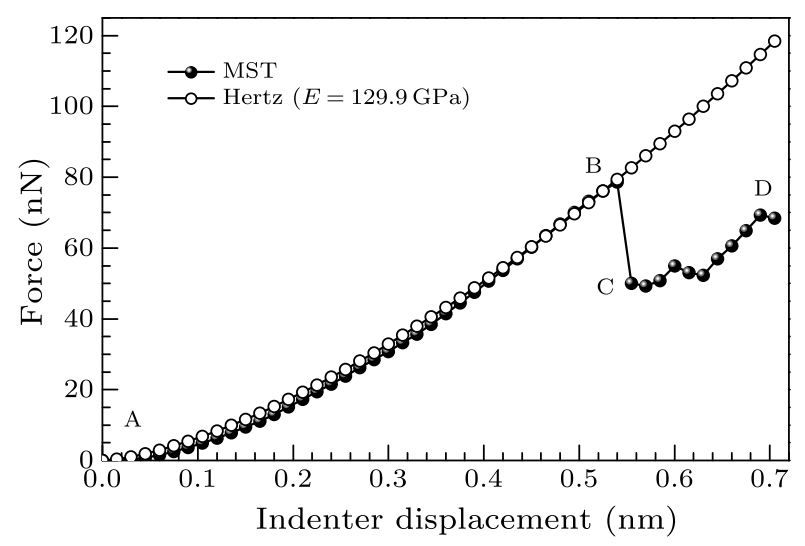

Fig. 1. Force vs indenter displacement for the (001) copper surface during the indentation of a spherical indenter. The hollow dots are the solution of the Hertzian elastic theory of contact with the fitted indentation modulus as 129.9 GPa, while the solid dots are the result of the MST simulation.

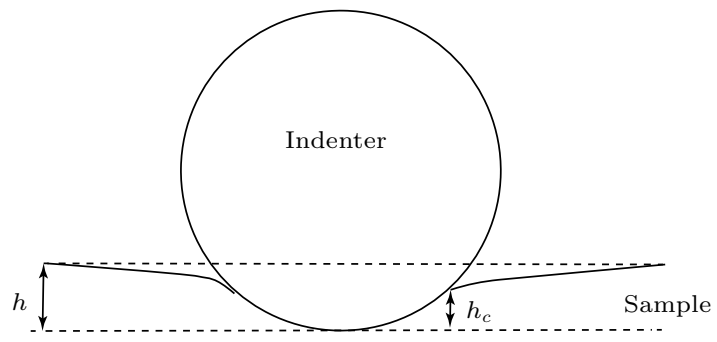

Fig. 2. Schematic diagram of the Hertzian elastic contact model. Here, $h$ is the maximum displacement of the indenter and $h_{c}$ is the contact depth.

In Hertzian elastic contact theory, the relationship between the maximum displacement of indenter, $h$, and the depth at the edge of contact, $h_{c}$, (Fig. 2$)$ is $h=2 h_{c}$. In the case of the MST simulation, the maximum displacement of the indenter is defined as the displacement of atoms in contact with the apex of the indenter, while the contact depth is defined as the displacement of atoms at the perimeter of the contact area. The $h_{c}$ data obtained in the MST simulation are denoted by the round solid dots in Fig. 3. Obviously, considering of the discrete nature of the simulation, 
the simulation data (denoted by hollow dots) in the elastic phase agree well with Hertzian elastic contact theory. After the nucleation of dislocations, the contact depth increases significantly because of the pileup effect.

According to Hertzian elastic contact theory, for a spherical indenter, the contact area $A_{c}$ is proportional to the indenter radius $R$ and the indenter displacement $h$ as ${ }^{[17]}$

$$
A_{c}=\pi R h \text {. }
$$

In our simulation, the contact area is taken as the area of the projected polygon with vertices at the centers of the peripheral contact atoms. Figure 4 shows the contact area vs indenter displacement. It is evident that the discrete nature of the contact zone introduces a discrete evolution of the contact area. In addition, the relationship between the contact area and the indenter depth qualitatively agrees with Eq. (7) before the nucleation of dislocations.

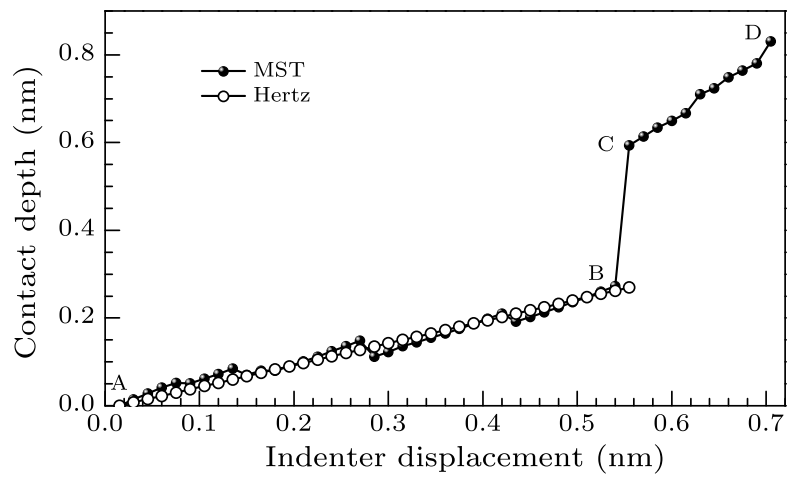

Fig. 3. Contact depth vs indenter displacement. The solid dots are the contact depths from the MST simulation, which are well described by Hertzian elastic contact solution (hollow) in the elastic regime. After dislocation nucleation, the contact depth in the MST simulation significantly increases, which indicates a pile-up situation.

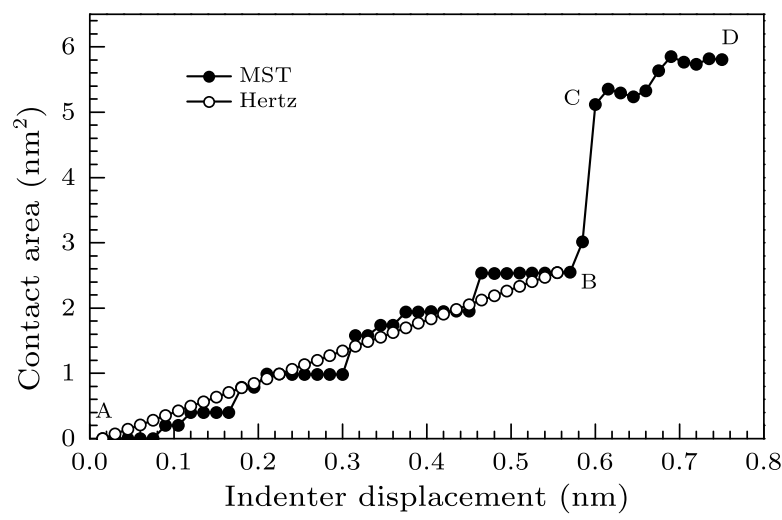

Fig. 4. Contact area versus indenter displacement. The solid dots are the data obtained from the MST simulation, and the hollow dots are the Hertzian elastic contact solution, calculated from the known tip geometry $(R=15 \AA)$ and indenter displacement.

Knowing the contact area $A_{c}$ and corresponding force $P$, we can calculate the mean pressure $p_{\text {mean }}$, which is equivalent to the indentation hardness $H$. This hardness characterizes the material's resistance to elastic and plastic deformation, and is defined as

$$
H=p_{\text {mean }}=P / A_{c} .
$$

Figure 5 demonstrates the variation of the mean pressure (indentation hardness) with the indenter displacement. The saw-tooth in the curve is due to the discrete evolution of the contact area with the discrete nature of the sample. It is shown that before the dislocation nucleation, the variation of mean pressure (hardness) vs indenter displacement quantitatively agrees with Hertizian elastic contact theory. The onset of plasticity causes the dramatic drop of hardness.

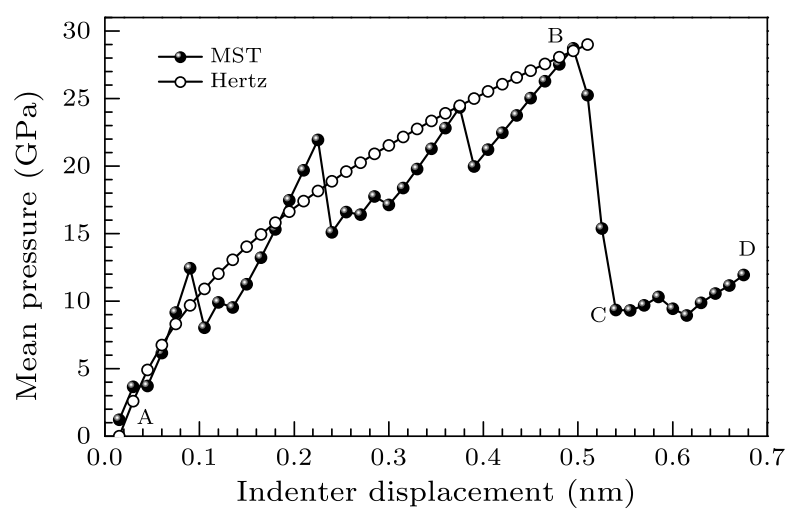

Fig. 5. Mean pressure vs indenter displacement. The line together with the solid dots are the data from the MST simulation. The hollow dots are the Hertzian elastic contact solution with a spherical indenter.

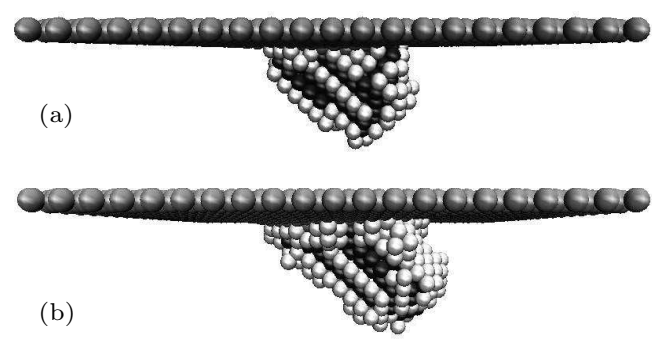

Fig. 6. Dislocation structure in the sample (a) at point $\mathrm{C}$ in Fig. 1, $h=0.56 \mathrm{~nm}$, and (b) at point D in Fig. 1, $h=0.71 \mathrm{~nm}$.

The dislocation structure in the sample can be investigated using the centro-symmetric parameter (CSP) method. ${ }^{[11]}$ Figure 6 shows the dislocation structure beneath the indenter from MST simulation. In Fig. 6, the atoms are colored according to its CSP value $Q$, with grey for surface atoms $(Q \geq 4.8)$; white for stacking faults $(1.6 \leq Q \leq 4.8)$; black for partial dislocation $(0.4 \leq Q \leq 1.6)$. Only atoms with $Q \geq 0.4$ are shown. Figure 6(a) shows the dislocation structure after slip at point $\mathrm{C}$ in Fig. 1, while Fig. 6(b) shows the dislocation structure at the maximmun indenter displacement at point D in Fig. 1. Obviously, slip occurs 
on four sets of (111) planes and terminates at (001) plane, and the (111) plane is the dominant slip plane in the FCC crystal. This is in good agreement with the early results obtained in the MD simulation. ${ }^{[11,18]}$

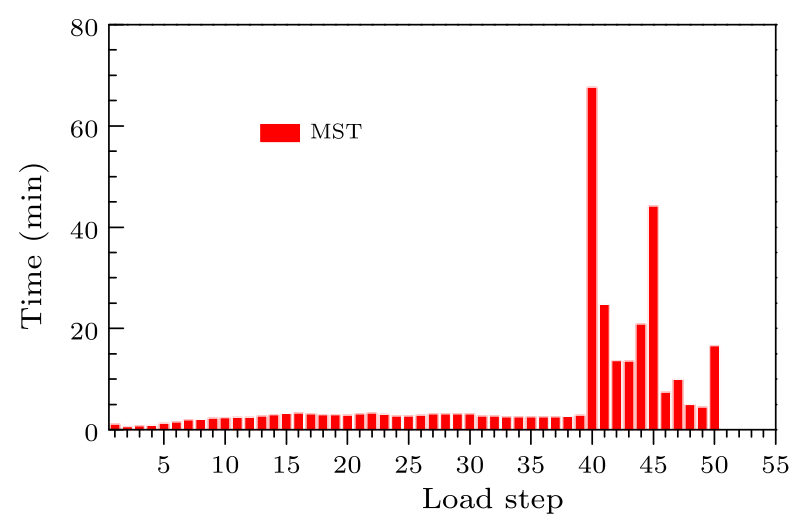

Fig. 7. The consuming time of each loading step in the MST simulation.

Lastly, let us compare the computation time for the indentation simulation by MST and the MD method. Figure 7 shows the consuming time in each loading step for the MST simulation. The several time-consuming loading steps correspond to the dislocation slips. The total computation time is $325 \mathrm{~min}$. For the same case, it takes 2405 min on the same PC with MD. Therefore, the neglection of atomic oscillation details in the MST simulation promotes the efficiency of the method by 8 times over that of the MD simulation.

In summary, the quasistatic nanoindentation process of a spherical indenter in single crystal copper is investigated with the MST method based on the EAM potential. The indentation modulus obtained in the MST simulation is $129.9 \mathrm{GPa}$, which agrees well with the theoretical data of $129 \mathrm{GPa}$. In the elastic regime, the obtained maximum displacement of the indenter is two times the contact depth, and the contact area is qualitatively proportional to the contact depth, which agrees well with the Hertzian elastic theory of contact. The MST simulation can reproduce the nucleation of dislocation as well. Moreover, the efficiency of the MST method is 8 times higher than that of traditional MD simulation.

\section{References}

[1] Oliver W C and Pharr G M 1992 J. Mate. Res. 71564

[2] Wang H Y, Bai Y L, Liu S, Wu J L and Wong C P 2002 Acta Mater. 504369

[3] Gannepalli A and Mallapragada S K 2001 Nanotechnology 12250

[4] Cheong W C D and Zhang L C 2000 Nanotechnology 11 173

[5] Leach A R 2001 Molecular Modeling Principles and Applications, 2nd edn (New Jersey: Prentice Hall)

[6] Wang H Y, Hu M, Bai Y L, Xia M F and Ke F J 2005 International Symposium on Multiscale Simulation (Hong Kong, China, December 2005)

[7] Wang H Y, Hu M, Xia M F, Ke F J and Bai Y L 2008 Int. J. Solids Struct. 453918

[8] Hu M 2002 PhD Dissertain (Beijing: Graduate University of Chinese Academy of Sciences)

[9] Born M and Huang K 1954 Dynamical Theory of Crystal Lattices (Oxford: Oxford University)

[10] McQuarrie D A 1976 Statistical Mechanics (New York: Harper \& Row)

[11] Kelchner C L, Plimpton S J and Hamilton J C 1998 Phys. Rev. B 5811085

[12] Daw M S and Baskes M J 1984 Phys. Rev. B 296443

[13] Mishin Y, Mehl M J, Papaconstantopoulos D A, Voter A F and Kress J D 2001 Phys. Rev. B 63224106

[14] Johnson K L 1985 Contact Mechanics (Cambridge: Cambridge University)

[15] Vlassak J J and Nix W D 1993 Philos. Mag. A 671045

[16] Vlassak J J and Nix W D 1994 J. Mech. Phys. Solids 42 1223

[17] Lilleodden E T, Zimmerman J A, Foiles S M and Nix W D 2003 J. Mech. Phys. Solids 51901

[18] Knap J and Ortiz M 2003 Phys. Rev. Lett. 90226102 